ARTICLE

Received 6 Nov 2013 | Accepted 2 Apr 2014 | Published 8 May 2014

DOI: $10.1038 /$ ncomms4774

OPEN

\title{
Bio-inspired detoxification using 3D-printed hydrogel nanocomposites
}

Maling Gou ${ }^{1,2, \star}$, Xin $\mathrm{Qu}^{3, \star}$, Wei Zhu ${ }^{3}$, Mingli Xiang ${ }^{1}$, Jun Yang ${ }^{4}$, Kang Zhang ${ }^{2,5,6}$, Yuquan Wei ${ }^{1} \&$ Shaochen Chen ${ }^{3,5}$

Rationally designed nanoparticles that can bind toxins show great promise for detoxification. However, the conventional intravenous administration of nanoparticles for detoxification often leads to nanoparticle accumulation in the liver, posing a risk of secondary poisoning especially in liver-failure patients. Here we present a liver-inspired three-dimensional (3D) detoxification device. This device is created by 3D printing of designer hydrogels with functional polydiacetylene nanoparticles installed in the hydrogel matrix. The nanoparticles can attract, capture and sense toxins, while the 3D matrix with a modified liver lobule microstructure allows toxins to be trapped efficiently. Our results show that the toxin solution completely loses its virulence after treatment using this biomimetic detoxification device. This work provides a proof-of-concept of detoxification by a 3D-printed biomimetic nanocomposite construct in hydrogel, and could lead to the development of alternative detoxification platforms.

\footnotetext{
${ }^{1}$ State Key Laboratory of Biotherapy and Cancer Center, West China Hospital, West China Medical School, Sichuan University, Chengdu 610041, P.R. China.

${ }^{2}$ Shiley Eye Center and Institute for Genomic Medicine, University of California, San Diego, La Jolla, California 92093, USA. ${ }^{3}$ Department of NanoEngineering, University of California, San Diego, La Jolla, California 92093, USA. ${ }^{4}$ School of Chemistry and Chemical Engineering, Shaanxi Normal University, Xi'an 710062, P.R. China. ${ }^{5}$ Biomaterials and Tissue Engineering Center, University of California, San Diego, La Jolla, California 92093, USA.

${ }^{6}$ Veterans Administration Healthcare System, San Diego, California 92093, USA. * These authors contributed equally to this work and are co-first authors. Correspondence and requests for materials should be addressed to M.G. (email: goumaling@scu.edu.cn) or to S.C. (email: chen168@eng.ucsd.edu).
} 
$\mathrm{O}$ wing to their inherent small size and flexibility in rational design and preparation, functional nanoparticles have shown potential applications in effective detoxification $^{1-6}$. Pore-forming toxins (PFTs) that can damage cellular membrane are key virulence factors of pathologies resulting from animal bites/stings and bacterial infections ${ }^{7-9}$. Conventional detoxification platforms such as antisera ${ }^{10}$, monoclonal antibodies ${ }^{11}$ and small-molecule inhibitors ${ }^{12}$ are hard to completely neutralize toxins because of their limited capability of blocking the whole PFTs' molecule. Meanwhile, over 80 PFTs have been identified, displaying diverse molecular structures and distinctive epitopic targets ${ }^{13}$. The commonly used antidotes target the specific molecular structures of PFTs; thus, customized treatments are required for different toxins. Recent advancements in the field have spurred the development of nanoparticles that can efficiently bind PFTs and neutralize their toxicity in vivo ${ }^{1,2,4}$. Although poisoned patients may benefit from these strategies, intravenous administration of nanoparticles causes nanoparticle-toxin accumulation in the liver ${ }^{1}$, thus leading to the risk of secondary poisoning especially in liverfailure patients ${ }^{14}$. Despite these challenges, cleaning blood by adsorption of toxins to retrievable nanoparticles provides an alternative strategy for detoxification. Meanwhile, similar to injection of antidotes, it is clinically approved to remove toxins by in vitro devices ${ }^{15}$.

In this work, we demonstrate that polydiacetylene (PDA) nanoparticles can attract, capture and sense PFTs. Taking advantages of three-dimensional (3D) biomimetic structure in enrichment, separation and detection ${ }^{16-18}$, we develop a bioinspired 3D detoxification device by installing PDA nanoparticles in a precise $3 \mathrm{D}$ matrix with modified liver lobule configuration via an advanced $3 \mathrm{D}$ printing technology, that is, dynamic optical projection stereolithography (DOPsL). The DOPsL technology utilizes a digital mirror array device (DMD) to generate dynamic photomasks that can be translated into a $3 \mathrm{D}$ complex structure through layer-by-layer photopolymerization of biomaterials. The DOPsL technology has great efficacy and versatility in fabricating 3D complex geometries for functional devices and even artificial tissues ${ }^{19-22}$. As schematically presented in Fig. 1, this bio-inspired $3 \mathrm{D}$ device is designed to efficiently collect and sense PFTs for future detoxification applications.

\section{Results}

Neutralizing toxin using PDA nanoparticles. To develop functional nanoparticles for constructing a 3D detoxification device, we used PDA nanoparticles derived from self-assembly of 10, 12-pentacosadiynoic acid (PCDA; Supplementary Fig. 1). Specifically, blue and colourless PDA nanoparticles with vesicle structure were prepared by ultraviolet irradiation of self-assembled colourless PCDA nanovesicles ${ }^{23,24}$. The nanoparticle surface is made of a $\pi$-conjugated polymer with alternating double- and triple-bond groups in the main polymer chain. The cell membrane-mimicking surface functions to attract, capture and neutralize toxins owing to the interactions between PDA and toxins. Binding toxins to PDA nanoparticles disrupts the extensively delocalized enyne backbones of molecularly ordered PDA side chains, thus inducing a fluorescence enhancement (none-to-fluorescence) as well as colour change (blue-to-red), as schematically illustrated in Fig. 2a.

We evaluated the ability of PDA nanoparticles to capture and neutralize toxins by a red blood cells' (RBCs) lysis test. A widely studied PFT, melittin, was chosen and mixed with PDA nanoparticles and then added to murine RBCs. The centrifuged RBCs' solution was incubated with normal saline (as a control) or melittin mixed with PDA nanoparticles at different concentrations, as shown in Fig. 2b. Qualitatively, melittin mixed with more PDA nanoparticles produced a clearer supernatant, indicating that less RBCs were damaged. The neutralization efficiency was quantified via colorimetric readings and is presented in Fig. 2c. The results show that PDA nanoparticles can capture and neutralize melittin in vitro. The melittin-binding capacity of PDA nanoparticles is higher than $1 \mathrm{mmol} \mathrm{g}^{-1}$. This capacity is greater than that of previously reported protein-adsorbing films ${ }^{25}$, nanofibres $^{26}$ and nanoparticles ${ }^{1}$. The neutralization efficiency of PDA nanoparticles is $\sim 92 \%$. We also studied the ability of PDA nanoparticles to sense toxins. As shown in Fig. 2d, the interaction between melittin and PDA significantly enhanced the red fluorescence of PDA nanoparticles. The red fluorescence intensity of PDA increased with the concentration of melittin. Meanwhile, the binding of melittin can also induce a slight colour change of PDA nanoparticles (see Supplementary Fig. 2). By using the fluorescence data, we calculated the binding constant between melittin and PDA nanoparticles as $630 \mathrm{M}^{-1}$ from Langmuir isotherm. Compared with recently reported nanoparticles with $100 \%$ neutralization of melittin ${ }^{1,4}$, the PDA nanoparticle has a lower binding constant, which may be one of the reasons why PDA nanoparticles cannot achieve 100\% neutralization of melittin.

To better understand the interaction between PDA nanoparticles and melittin, we performed computer simulations at the molecular level. The optimized structure of PDA is present in Supplementary Fig. 3. After docking PDA to melittin, nine conformations of complex composed of melittin and PDA were obtained. For all of these conformations, the binding affinities between the two components of the complex are negative, suggesting that PDA possesses the capability to bind melittin. The conformation on the top of the list (Fig. 3a, I) has the most negative binding affinity: $-1.8 \mathrm{kcal} \mathrm{mol}^{-1}$. In this conformation, PDA interacts with melittin via its hydrophobic tails (seven, in total) and one polar head (Fig. 3a, II). On the other hand, half of 26 residues in melittin interact with PDA. Among these residues, only Thr10, Lys21 and Arg24 are polar residues (Fig. 3a, III). An electrostatic interaction between the carboxyl group of one polar head in PDA and the guanidine part of Arg24 in melittin was also observed (Fig. 3a, I). The interactions between PDA and melittin were further studied by performing simulated annealing using molecular dynamics (MD). MD simulation can facilitate modelled system to surmount small energy barriers on the potential surface and locate sites of lower potential energy, while

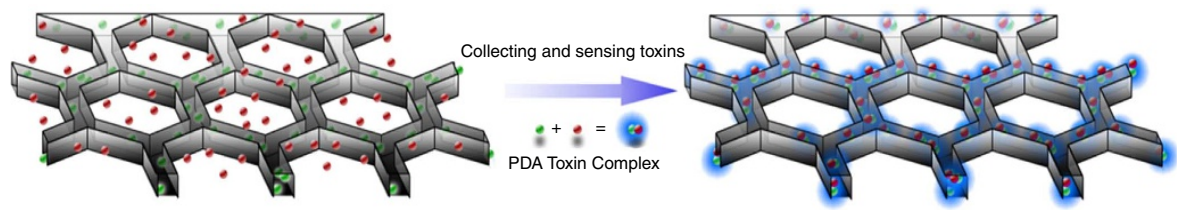

Figure 1 | Bio-inspired 3D detoxification device. PDA nanoparticles (green) are installed in PEGDA hydrogel matrix (grey) with liver-mimetic 3D structure fabricated by 3D printing. The nanoparticles attract, capture and sense toxins (red), while the 3D matrix with modified liver lobule structure allows toxins to be trapped efficiently. This biomimetic 3D detoxifier has promising clinical application for detoxification by collecting and removing toxins. 
a

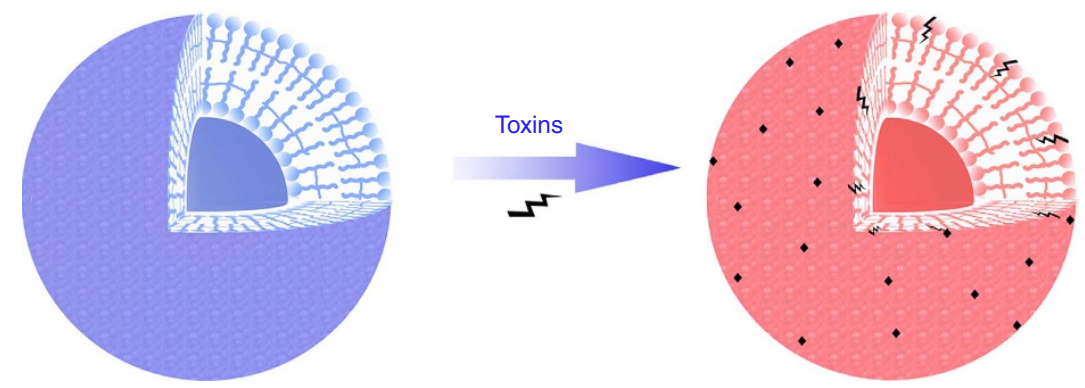

b

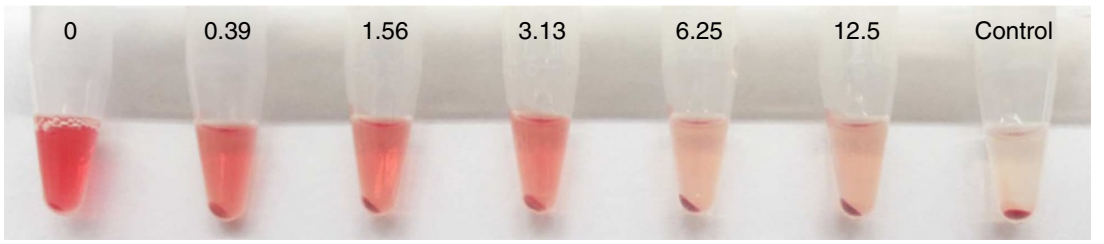

C
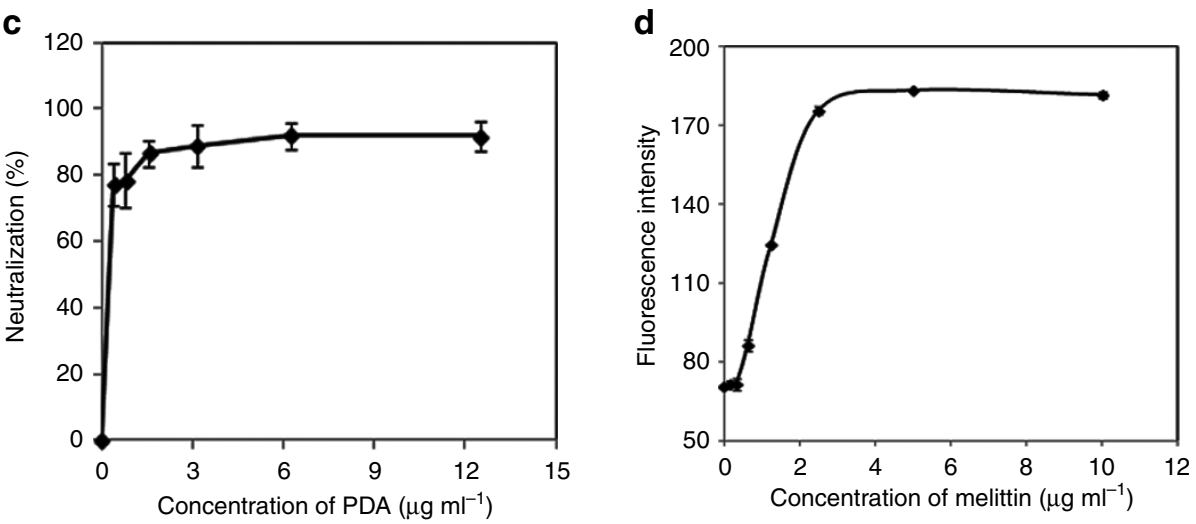

Figure 2 | Neutralization of toxins by PDA nanoparticles. (a) Schematic presentation of the interaction between PDA nanoparticles and toxins. PDA nanoparticles with cellular membrane-mimetic surface can attract and capture toxins, accompanied with fluorescence change (none-to-fluorescent). (b) Centrifuged RBCs after incubation with normal saline (control) or melittin $\left(5 \mu \mathrm{g} \mathrm{ml}^{-1}\right.$ ) mixed with different concentrations of PDA nanoparticles. (c) Quantified efficiency of neutralizing the haemolytic activity of melittin $\left(5 \mu \mathrm{g} \mathrm{ml}^{-1}\right)$ by PDA nanoparticles. (d) Intensity of red fluorescence of PDA nanoparticles $\left(20 \mu \mathrm{g} \mathrm{ml}^{-1}\right)$ that were exposed to melittin with different concentrations at $37^{\circ} \mathrm{C}$ for $30 \mathrm{~min}$.

dynamic annealing can obtain a minimum energy. As exhibited in Fig. 3b, PDA and melittin adjusted their orientations and approached each other in the simulation, suggesting that PDA can attract melittin in close proximity, followed by capture. Also, we found that interaction with melittin can affect the conformation of PDA, which may explain our experimental findings of fluorescence enhancement and colour change of PDA after incubation with melittin. The last conformation of the modelled system was experiencing simulated annealing and subsequent equilibrium. Moreover, by simulated annealing using MD, the PDA-melittin interaction mode was obtained and presented in Fig. 3c. On the basis of these simulations, we conclude that hydrophobic and electrostatic interactions exist between PDA and melittin, and the former interaction contributes much more than the latter; this in theory supports PDA nanoparticles to capture PFTs.

Capturing toxin in the 3D-printed hydrogel nanocomposites. After confirming the PDA nanoparticles' ability to attract and capture melittin in solution, we designed a $3 \mathrm{D}$ device consisting of a biomaterial hydrogel scaffold to make the nanoparticles retrievable. We used a photocrosslinked poly(ethylene glycol) diacrylate (PEGDA) hydrogel as a $3 \mathrm{D}$ matrix. PEGDA is often used in biomedical applications because it is non-toxic, non-immunogenic, favourable to nutrient and oxygen transport, and tunable in its mechanical properties. To chemically link PDA nanoparticles into the networks of PEGDA hydrogel, we synthesized a diacetylene derivative called PCDA-acrylamide (PCDA-A 5, Supplementary Fig. 4). It is based on the diacetylene moiety of PCDA with an extended ethylene oxide spacer arm and an acrylamide functional head group. By mixing PCDA and PCDA-A 5, the resulting nanoparticles possess acrylamide group on its surface (Supplementary Fig. 5a), and can be chemically tethered to the PEGDA hydrogel through addition polymerization (Fig. 4a). The nanoparticles have a mean particle size of $\sim 100 \mathrm{~nm}$ according to the scanning electron microscope image (Supplementary Fig. 5b). The particles' distribution was determined by dynamic light scattering, as shown in Supplementary Fig. 6a. The nanoparticles have a mean particle size of $110 \mathrm{~nm}$ with polydispersity index of 0.23 . Meanwhile, the nanoparticles have a zeta potential of $-25 \mathrm{mV}$ (Supplementary Fig. 6b). Owing to the extremely slow degradation rate of PEGDA network, once chemically immobilized in PEGDA hydrogel, it is difficult for PDA nanoparticles to release into the solution. As shown in Supplementary Fig. 7, no significant amount of PDA nanoparticles were released from the PEGDA hydrogel in $24 \mathrm{~h}$.

Taking advantages of the photocrosslinkable hydrogel, DOPsL technology, which employs a digital mask and ultraviolet light source for layer-by-layer photopolymerization, was utilized to 
a
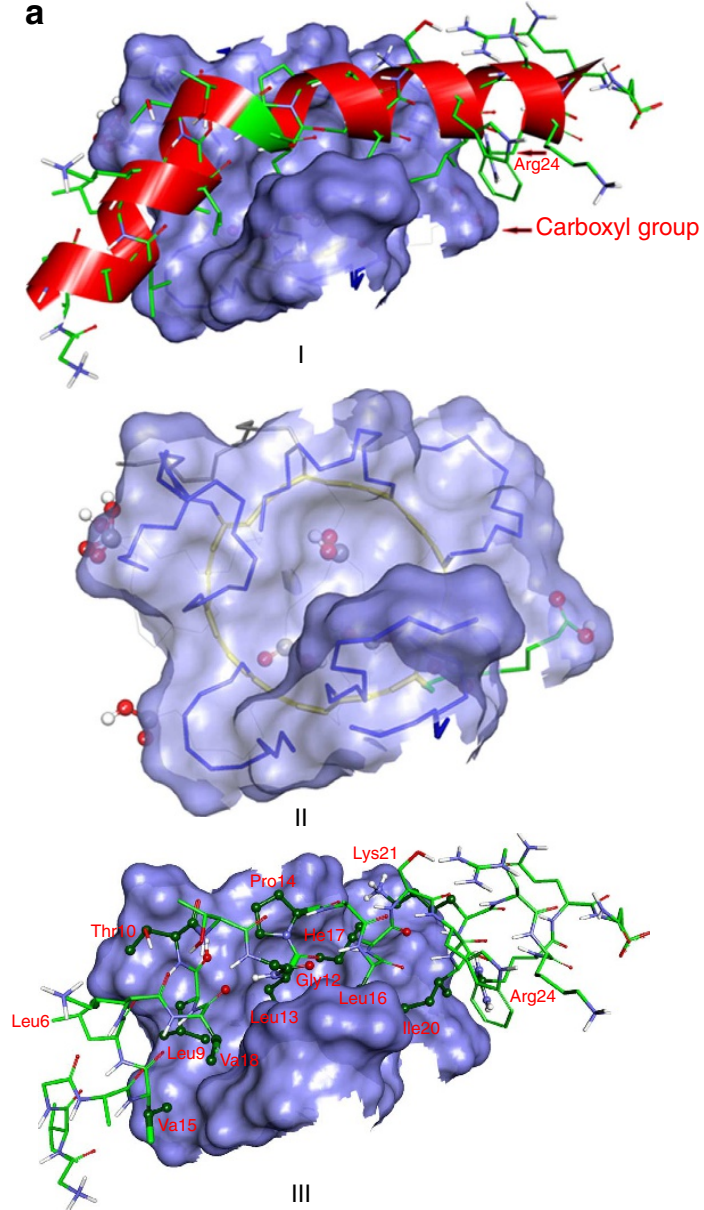

b
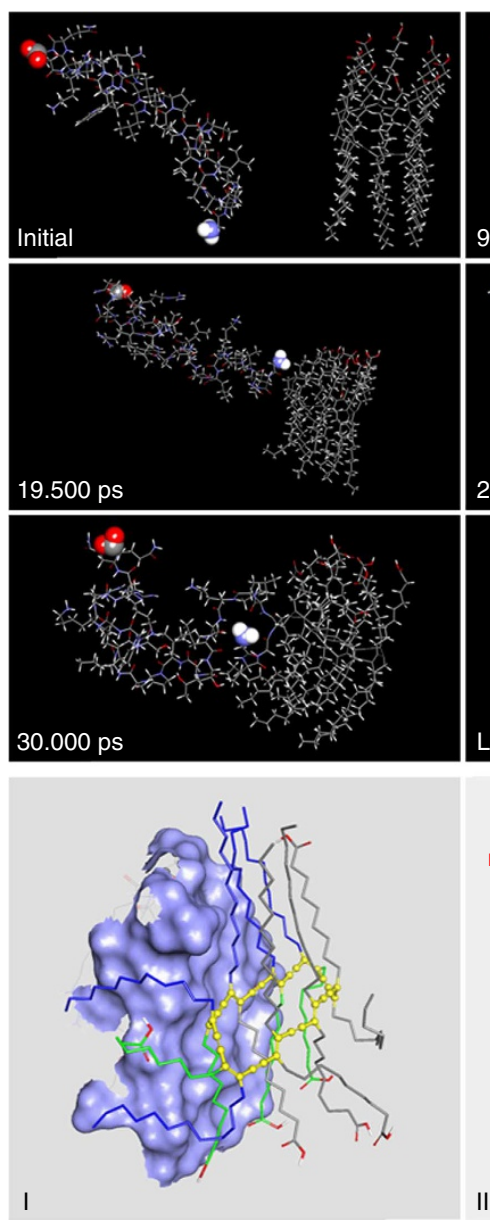

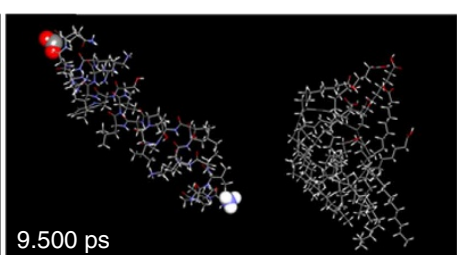

$9.500 \mathrm{ps}$
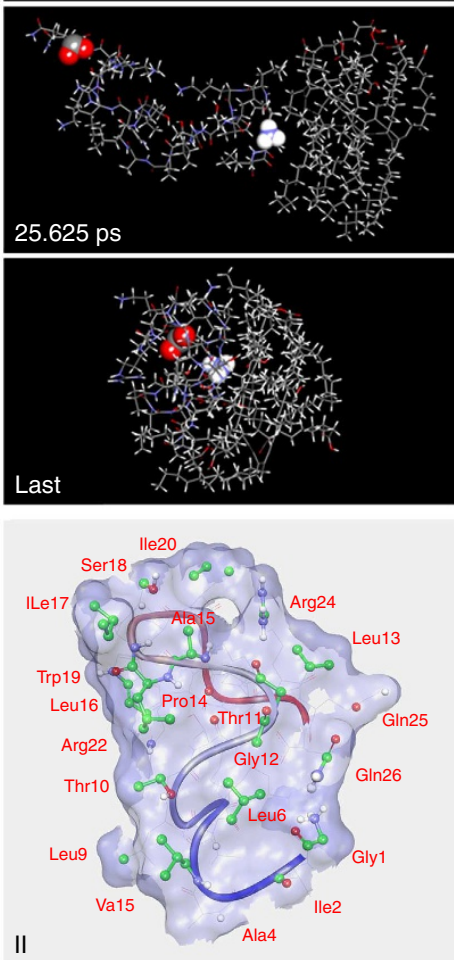

Figure 3 | Computer simulations on the interactions between PDA and melittin. (a) Simulation results obtained by docking PDA to melittin.

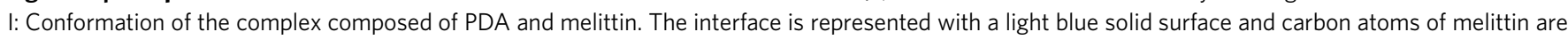
coloured with green. Groups that take part in electrostatic interaction have been marked. II: PDA under the interface. Polar heads and hydrophobic tails that interact with melittin are represented with stick. Their carbon atoms are coloured with green and blue, respectively. III: Melittin above the interface. Atoms that interact with PDA are represented with scaled ball and stick and carbon atoms are coloured with dark green. (b) Interactions between PDA and melittin studied by performing simulated annealing using MD. PDA and melittin adjust their orientations and approach each other, followed by forming a complex. The carboxy terminal and amino terminal of melittin are highlighted. (c) PDA-melittin interaction mode obtained by performing simulated annealing using MD. I: PDA on the interaction interface. Polar heads and hydrophobic tails that interact with melittin are represented with stick and

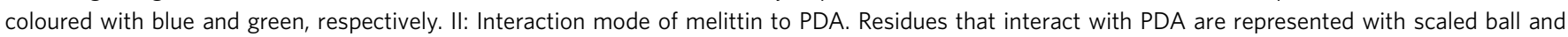
stick and their carbon atoms are coloured with green.

fabricate 3D PEGDA hydrogels with precise microstructures, as shown in Fig. 4b. As a proof of concept, PEGDA hydrogels with and without PDA nanoparticles were fabricated into simple 3D cylinders and subsequently incubated with melittin solution $\left(50 \mu \mathrm{g} \mathrm{ml}^{-1}\right)$. As shown in Fig. $4 \mathrm{c}$, initially the control PEGDA hydrogel (without nanoparticles) was transparent and nonfluorescent. No colour or fluorescent changes were detected after incubation with melittin. In comparison, PDA nanoparticles incorporated hydrogel appeared opaque (the colour is blue because of the PDA) without fluorescence initially. After incubation with melittin solution $\left(50 \mu \mathrm{g} \mathrm{ml}^{-1}\right)$, however, the composite hydrogel cylinder emanated red fluorescence, which gradually diffused from the surface to the inner part in a time-dependent manner, indicating successful binding of melittin to PDA.

Next, we studied the effect of the hydrogel's specific surface area on the efficiency of capturing toxins. We designed and fabricated three types of 3D posts with the same flower-like projection and length but different diameters, where the thinner post displayed higher specific surface area. As shown in Fig. 5, melittin can gradually diffuse into all types of post. In the same period, red fluorescence can only be detected on the outer area of the thicker posts while in the thinner posts, the fluorescent signals were also observed in the inner region. On the basis of the previous data that the fluorescent signals correspond to PDA-melittin interaction, the fluorescent signal trends we observed here could imply a modulation of PDA-melittin interactions owing to the specific surface area. The $3 \mathrm{D}$ structure containing PDA nanoparticles with a higher specific surface area may be more efficient in enriching toxins. In addition, we found that macromolecules with a higher molecular weight diffuse slower into the PEGDA hydrogel (as shown in Supplementary Fig. 8), implying that physical properties such as the porosity of the hydrogel can affect the diffusion of molecules into PEGDA hydrogel, and the PEGDA hydrogel may allow melittin to diffuse faster into the inner structure compared with others macromolecular proteins in the plasma. 
a

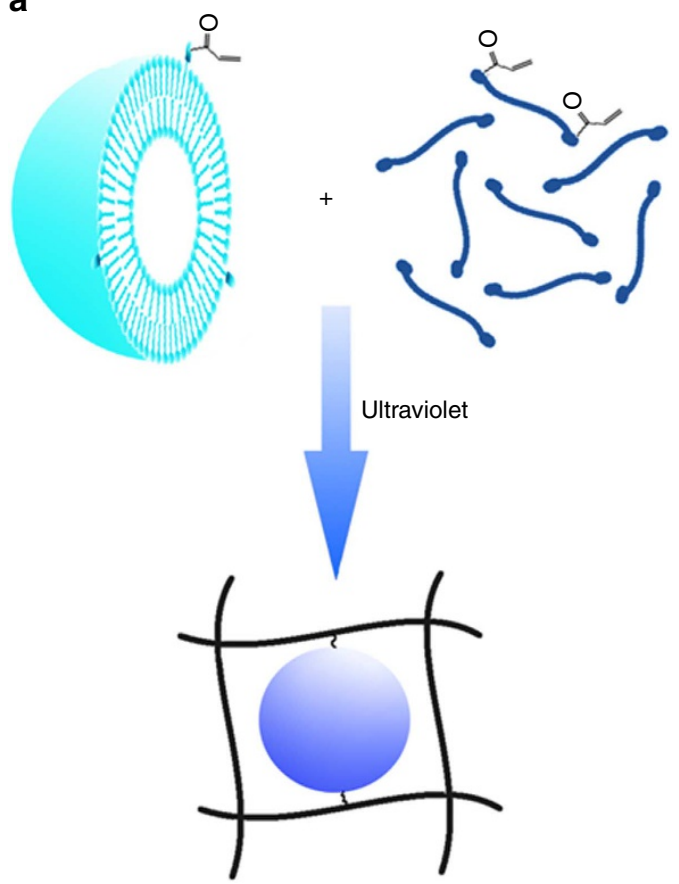

b

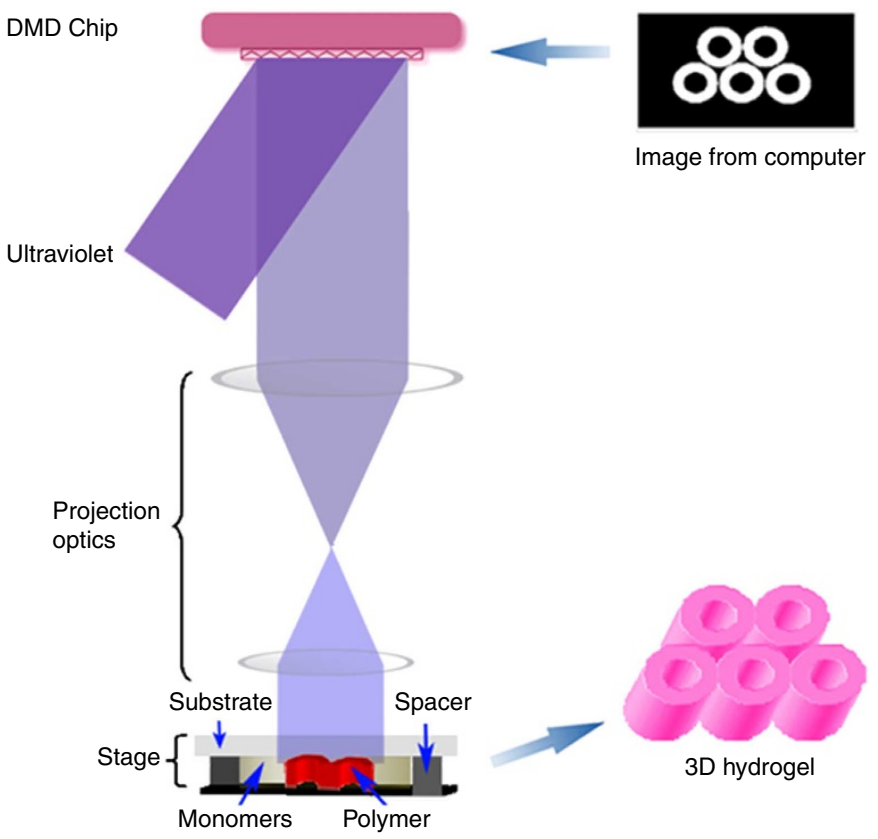

c
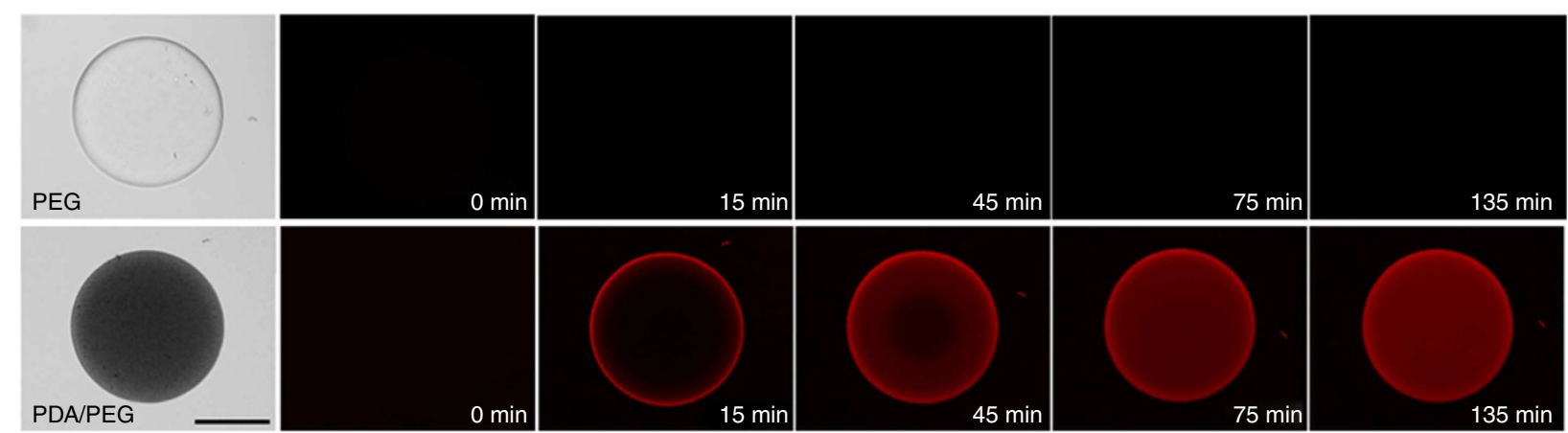

Figure 4 | Immobilization of PDA nanoparticles in 3D-structured PEGDA hydrogel. (a) Schematic presentation of installing PDA nanoparticles in the network of PEGDA hydrogel. PDA nanoparticles can be chemically linked to the network of PEGDA hydrogel by photocrosslinking PEGDA monomer and acrylamide-modified PDA nanoparticles via addition polymerization. (b) Schematic drawing of the DOPsL method to fabricate 3D-structured PEGDA hydrogel. By rationally designing the masks, PEGDA hydrogel with precise 3D structure can be created. (c) The fluorescence of PEGDA hydrogel of cylindrical shape with or without PDA nanoparticles after incubation with a melittin solution $\left(50 \mu \mathrm{g} \mathrm{ml}^{-1}\right)$. The fluorescence indicates that melittin is captured by PDA nanoparticles. Scale bar, $50 \mu \mathrm{m}$.

Designing the bio-inspired detoxification device. To move this surface area-dependent detoxification material to a physiologically relevant design, we integrated the structure of the liver, given its utility in the body's detoxification. The liver has a hexagonal lobule structure centred around the terminal hepatic vein, which helps to efficiently remove wastes and xenobiotics from the system. We therefore designed a liver-mimetic structure with modified liver lobule topology that allows the toxin to reach the centre of the matrix quickly, as shown in Fig. 6a. Our modified structure has a higher specific surface area than the typical liver lobule structure, which is beneficial for toxins to enter the $3 \mathrm{D}$ device effectively. Two types of mask patterns (Fig. 6b) were designed and alternatively printed by the DOPsL method layerby-layer, and finally a liver-mimetic $3 \mathrm{D}$ detoxifier was fabricated. After incubated with melittin $\left(50 \mu \mathrm{g} \mathrm{ml}^{-1}\right)$, the $3 \mathrm{D}$ microstructure of this detoxifier was observed under a confocal microscope. From the red fluorescence of toxin-bound PDA nanoparticles, the structure of this detoxifier was reconstructed and the results are presented in Fig. 6c. A scanning electron microscope image revealed the multilayer microstructure with modified liver lobule topology (Fig. 6d). Meanwhile, we can observe the surface of the matrix with some arrested nanoparticles (Supplementary Fig. 9). Finally, we tested the capability of this detoxifier to capture toxins. Compared with the slab, the liver-mimetic structure makes the detoxifier to neutralize toxins faster, owing to the faster diffusion of toxins into the inner structure (Fig. 6e). In Fig. 6f, the RBC solution incubated with melittin treated by our biomimetic detoxifier has a clear supernatant similar to that of the saline control. The red colour of the supernatant in PDA nanoparticle-treated group is more significant than that in the biomimetic detoxifier-treated group. Our 3D biomimetic detoxifier achieved 100\% neutralization of toxins while the equivalent amount of free PDA nanoparticles neutralizes $91 \%$ toxins, and the PEGDA hydrogel matrix alone has little capacity in neutralizing toxins (shown in Fig. 6g). Therefore, this biomimetic detoxifier can effectively neutralize toxins, serving as a new technology platform for detoxification. 


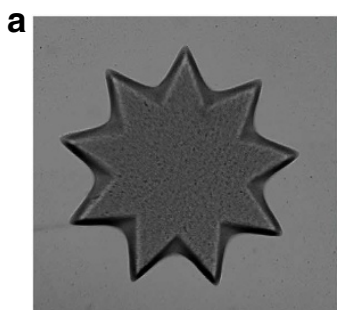

b

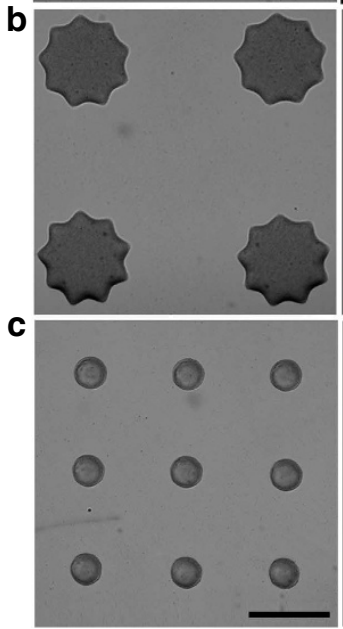

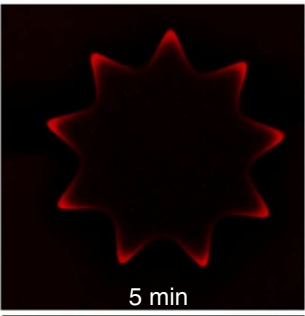
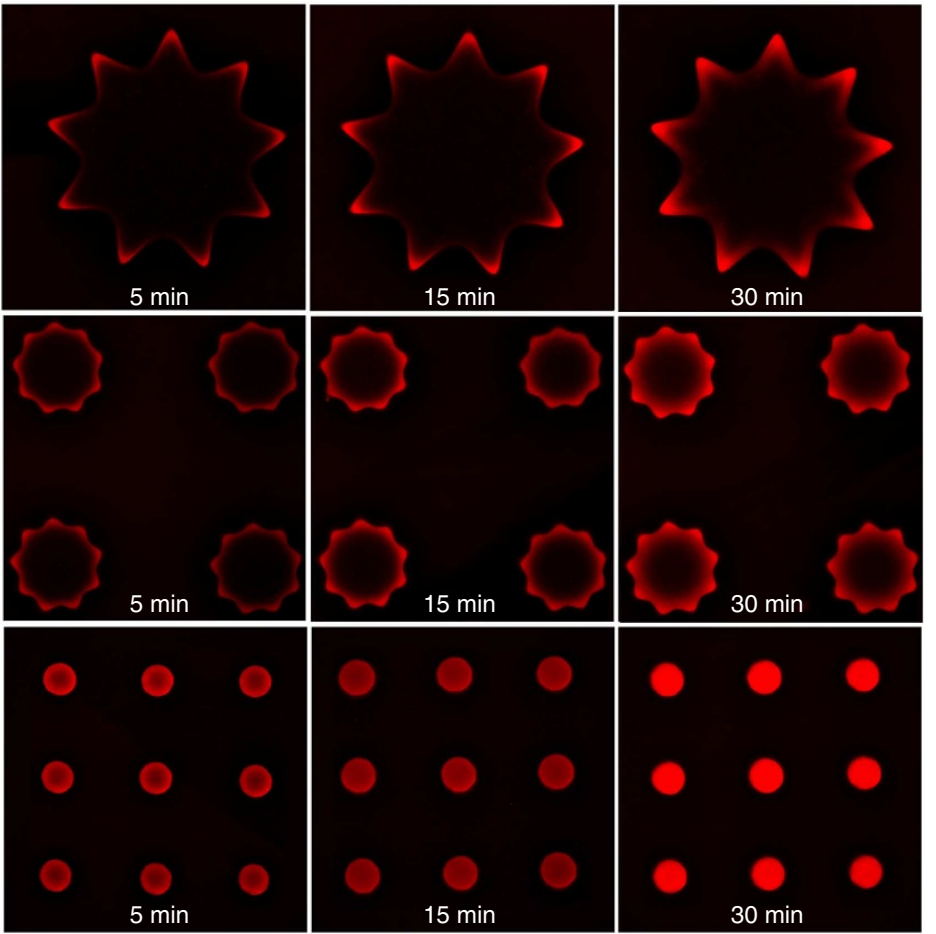

Figure 5 | Capturing toxin in the 3D-printed hydrogel nanocomposites with different surface patterns. Three types of 3D structural posts with the same flower-like shape and length but different diameters are incubated with melittin solution $\left(50 \mu \mathrm{g} \mathrm{ml}-1\right.$ ) for different time at $37^{\circ} \mathrm{C}$. (a) The post with a large diameter. (b) The post with a medium diameter. (c) The post with a small diameter. The red fluorescence implies the PDA and melittin interactions, indicating the site that toxins reach at. Scale bar, $200 \mu \mathrm{m}$.

\section{Discussion}

PDA is a $\pi$-conjugated polymer that has been extensively studied as a sensor matrix, because of its colour (blue-to-red) and fluorescence (none-to-fluorescent) changes in response to environmental cues ${ }^{27-32}$. Currently, while synthesis and utilization of DA monomers with new functional groups remains interesting ${ }^{33}$, expanding their utility as functional building blocks to construct 3D supermolecular assemblies enables new and improved applications of PDAs ${ }^{34,35}$. 3D PDAs show superior properties such as higher stability, increased surface area and multipoint interaction sites. In this work, a new application of PDA nanoparticles for detoxification was demonstrated. We used computer simulations to understand the molecular mechanism of interaction between PDA and melittin. Moreover, we synthesized PCDA-A 5 functional nanoparticles with alkenyl groups on its surface, followed by chemical immobilization in a photocrosslinked 3D hydrogel using $3 \mathrm{D}$ printing. To our knowledge, this is the first attempt to precisely incorporate PDAs in $3 \mathrm{D}$ hydrogels with a biomimetic architecture.

We specifically used PEG-derived hydrogels for the 3D detoxification device. PEG is well known as a biocompatible and 'stealth' material ${ }^{36,37}$, and PEG hydrogels have been widely used in biomedical applications such as tissue engineering ${ }^{38}$, drug delivery $^{39}$ and in vitro $3 \mathrm{D}$ cell culture ${ }^{40,41}$. In addition, the pore sizes of the patterned PEG hydrogel can be easily tuned to enable efficient solute exchange ${ }^{42}$ and PEG itself is resistant to biofouling and non-specific absorption of biomacromolecules in vitro and in vivo ${ }^{43}$. Therefore, PEG hydrogel is an ideal biocompatible matrix for carrying functional nanoparticles, allowing the nanoparticles to function in a controlled 3D space ${ }^{44}$.

In this work, PDA nanoparticles are chemically immobilized in a 3D PEG hydrogel and used for collecting and sensing toxins.
This idea of incorporating particles into hydrogel for detoxification purposes has been demonstrated by early studies ${ }^{45-47}$. We propose to shape the PEG matrix encapsulated with PDA nanoparticles into a designer structure using $3 \mathrm{D}$ printing, a disruptive technology that is finding promising applications in creating unique $3 \mathrm{D}$ devices and tissue constructs ${ }^{48,49}$. Inspired by the liver structure that contributes to fast substances' exchange between blood stream and hepatic cells ${ }^{50}$, we fabricated PEG hydrogels into a liver-mimetic structure with modified liver lobule configuration, producing a higher specific surface area than the original liver lobule. This bio-inspired designer 3D structure allows toxins to enter the PEG matrix effectively and interact with these nanoparticles with toxins' affinity, providing new insight for rationally designing a $3 \mathrm{D}$ detoxifier for potential clinical applications in detoxification ex vivo.

PFTs are one of the most common peptide toxins. Recently, based on the functional similarity among different PFTs in perforating cellular membranes, an action mechanism-targeted detoxification concept was illustrated ${ }^{2}$, suggesting that cellular membrane-mimetic matrix can be used to capture and neutralize a variety of PFTs. In our work, PDA nanoparticles with a cellular membrane-mimetic surface are developed to attract, capture and sense toxins, followed by immobilization within a liver-mimetic 3D PEG hydrogel matrix, creating a 3D biomimetic detoxifier. Our data show that the toxin's solution completely lost its virulence in damaging cellular membrane after treatment with the $3 \mathrm{D}$ detoxifier. Recently, Hoshino et al. ${ }^{1,4}$ prepared a library of tailored multifunctional copolymer nanoparticles and demonstrated that their nanoparticles with high binding affinity can achieve $100 \%$ neutralization of toxins in vitro, providing a novel method for designing advanced plastic antidotes. Similar to other cellular membrane-mimetic nanoparticles ${ }^{2}$, the PDA nanoparticles can work as artificial cells to efficiently neutralize 
a
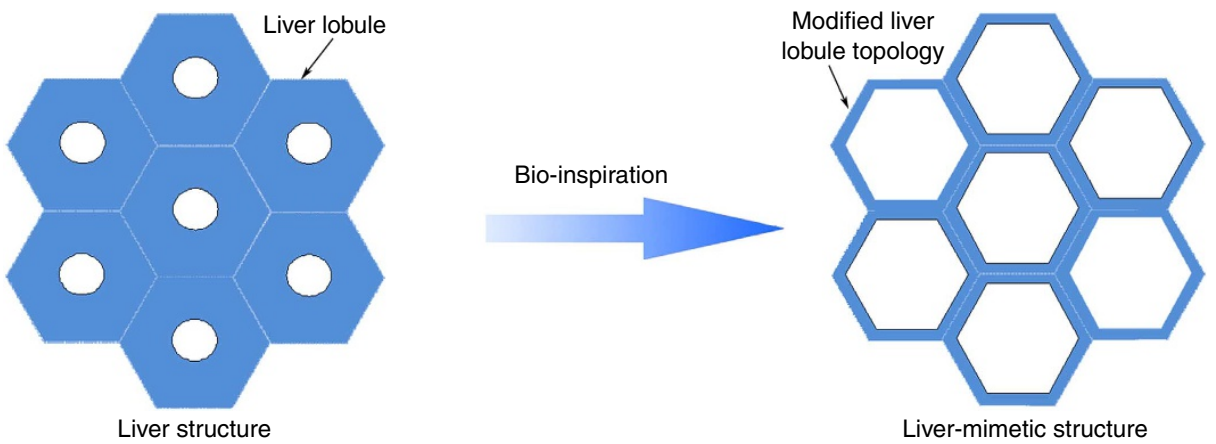

b
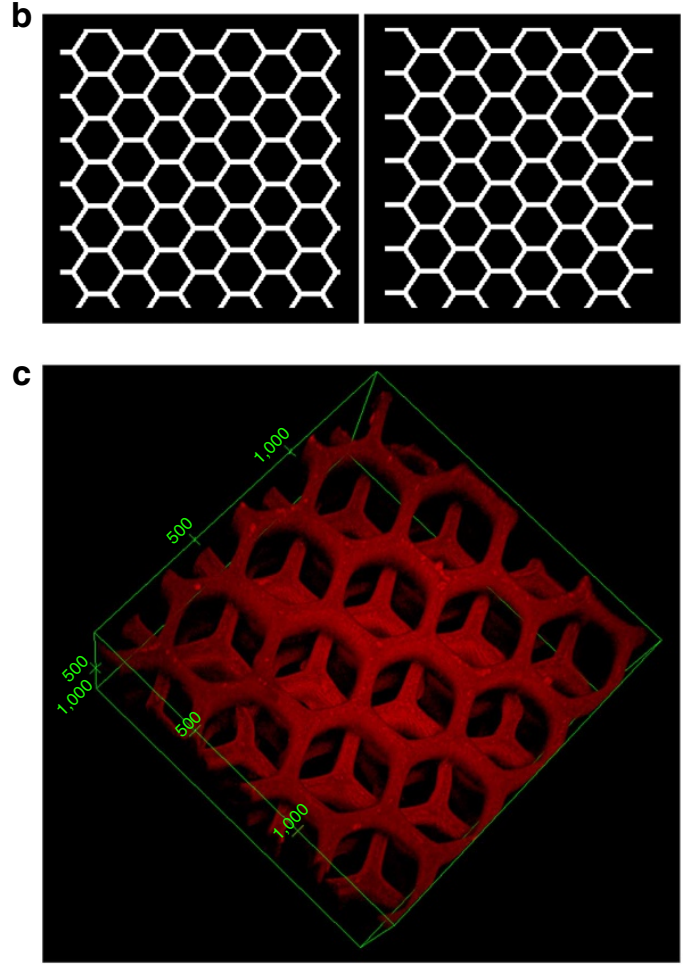

f
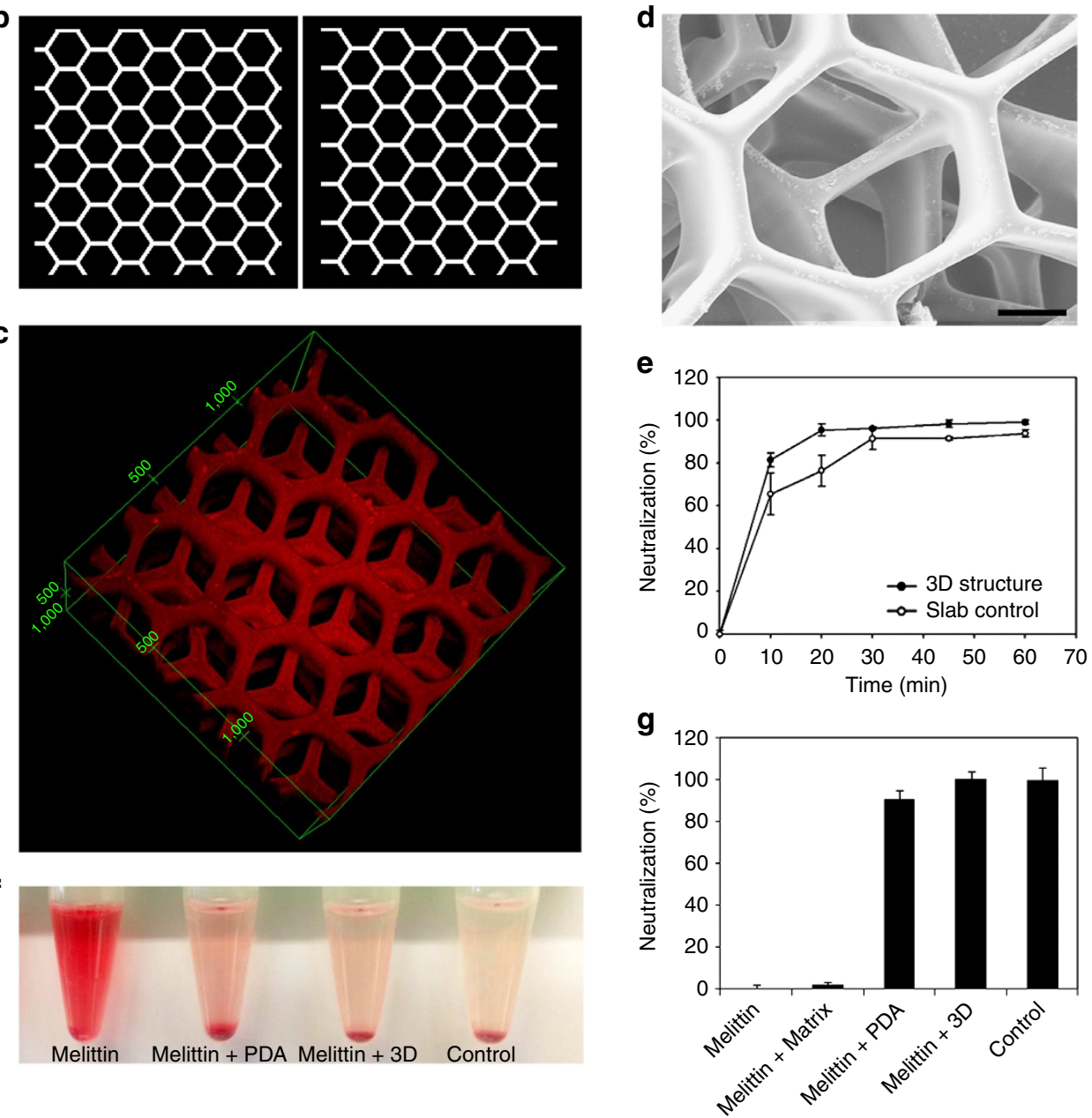

Figure 6 | The bio-inspired 3D detoxifier. (a) Rational design of the liver-mimetic 3D structure. The modified liver lobule topology allows toxins to enter the 3D matrix efficiently. (b) The masks that are used for printing liver-mimetic 3D structure. By alternatively using these masks in DOPsL technology, a bio-inspired detoxifier with four layers of liver-mimetic structure is created. (c) The 3D structure of the detoxifier measured by laser confocal microscopy. Incubated with melittin $\left(50 \mu \mathrm{g} \mathrm{ml}^{-1}, 300 \mu \mathrm{l}\right)$, the red fluorescence of PDA allows the reconstruction of the microstructure of the detoxifier in 3D.

(d) Scanning electron microscope image of this detoxifier. Scale bar, $50 \mu \mathrm{m}$. (e) Dynamic test on the neutralization efficiency with comparison between the liver-mimetic structure (3D structure) and slab control (that is, with the same total volume). After the incubation of melittin solution with detoxifier for different times, the neutralization efficiency was tested by the haemolytic assay. (f) Centrifuged RBCs after incubation with normal saline (control), melittin $\left(5 \mu \mathrm{g} \mathrm{ml}^{-1}\right)$, detoxifier-treated melittin or PDA nanoparticles-treated melittin. The red colour indicates the cytolysis of RBCs. (g) Neutralization efficiency of the 3D detoxifier. Equivalent amount of PDA nanoparticles is used for comparison.

PFTs, although they cannot achieve $100 \%$ neutralization efficiency, possibly because of a low binding constant. Our 3D detoxifier can separate the toxin/PDA complex from cells so that the RBCs are limited to interact with the melittin on the surface of PDA nanoparticles, resulting in an enhanced detoxification efficiency compared with free PDA nanoparticles. In addition, conventional detoxification in intravenous application of nanoparticles shows risk of causing secondary poisoning especially in liver-failure patients, owing to their accumulation in the liver ${ }^{1,2}$. Here inspired by the liver microstructure, we demonstrate a liver-mimetic 3D detoxification device that can collect and remove toxins, providing a novel strategy for designing nanoparticle-enabled detoxification treatments. Moreover, the cell-free nature of our detoxifier allows 
integration of a variety of functionalities and nanoelements in rationally designed microarchitectures. This could lead to many breakthroughs in the development of future detoxification platforms.

\section{Methods}

Materials. PEGDA $(\mathrm{Mw}=700 \mathrm{Da})$, melittin and PCDA were purchased from Sigma (USA). PCDA was further purified by dissolving in dichloromethane and then filtered to remove polymerized monomers before use. Photoinitiator lithium phenyl-2,4,6-trimethylbenzoylphosphinate was synthesized according to the previous work ${ }^{22}$

Computational simulation. The interactions between melittin and PDA were studied using two kinds of computational simulation methods. One is to dock PDA to melittin with the help of AutoDock Vina and the other is to perform simulated annealing using MD (Supplementary Methods).

Synthesis and identification of PCDA-A 5. The synthesis scheme of PCDA-A 5 has been presented in Supplementary Fig. 4a. In a 50-ml flask with a stir bar, compound 1 (PCDA, $374 \mathrm{mg}, 1.0 \mathrm{mmol}$ ) was dissolved in dry $\mathrm{CH}_{2} \mathrm{Cl}_{2}(10 \mathrm{ml})$ followed by addition of triethylamine $(101 \mathrm{mg}, 1.0 \mathrm{mmol})$ and $N, N^{\prime}$-disuccinimidyl carbonate $(256 \mathrm{mg}, 1.0 \mathrm{mmol})$. The reaction solution was stirred at room temperature for $1 \mathrm{~h}$ to activate compound $\mathbf{1}$, creating active ester $\mathbf{2}$. Then, acid 3 (74 mg, $1.0 \mathrm{mmol}$ ) was dissolved in dry $\mathrm{CH}_{2} \mathrm{Cl}_{2}(10 \mathrm{ml})$, followed by adding triethylamine $(101 \mathrm{mg}, 1.0 \mathrm{mmol})$ and $N, N^{\prime}$-disuccinimidyl carbonate $(256 \mathrm{mg}$, $1.0 \mathrm{mmol})$. The resulting solution was stirred at room temperature for $1 \mathrm{~h}$, and then amine 4 was added. The reaction solution was monitored by liquid chromatography-mass spectrometry. Four hours later, ester 2 was added. The reaction was stirred at room temperature for $4 \mathrm{~h}$, product 5 (PCDA-A 5) was found by liquid chromatography-mass spectrometry analysis. The reaction solution was evaporated and the residue was purified by flash column chromatography to produce $84 \mathrm{mg}$ compound $\mathbf{5}$ as white solid. The yield was 15\%. The PCDA-A $\mathbf{5}$ was identified by ${ }^{1} \mathrm{H}$ NMR (Supplementary Fig. 10), ${ }^{13} \mathrm{C}$ NMR (Supplementary Fig. 11) and high resolution mass spectrometer (Supplementary Fig. 12).

Preparation of PDA nanoparticles. To prepare the PDA nanoparticles, $40 \mathrm{mg}$ of PCDA was added into $8 \mathrm{ml}$ of distilled water, followed by probe sonication for 5 min at $\sim 75^{\circ} \mathrm{C}$. Then, the solution was stored overnight at $4{ }^{\circ} \mathrm{C}$. At last, the solution was irradiated with ultraviolet for $5 \mathrm{~min}$ in ice bath, creating blue and nonfluorescence PDA nanoparticles ${ }^{23}$. PCDA-A 5 and PCDA mixed nanoparticles were prepared by sonication of the mixed PCDA-A 5 and PCDA (mass ratio 1:19) in hot water (at about $75^{\circ} \mathrm{C}$ ).

3D printing via DOPsL. The DOPsL set-up illustrated in Fig. 4b was used to fabricate the $3 \mathrm{D}$ structures using photopolymerizable polymers ${ }^{21}$. The sliced images of the $3 \mathrm{D}$ model were loaded to the DMD chip that can continuously generate optical patterns and expose the photopolymerizable material in a layer-bylayer manner. PEGDA (20 wt\%) in $\mathrm{H}_{2} \mathrm{O}$ with $1 \%$ lithium phenyl-2,4,6trimethylbenzoylphosphinate was mixed with PDA particles suspension $\left(5 \mathrm{mg} \mathrm{ml}^{-1}\right)$ in an equal volume ratio. The mixture was then loaded to the sample stage of DOPsL and polymerized via patterned ultraviolet exposure to fabricate corresponding 3D structures. The DMD chip used in the set-up is DLP-07 XGA from DLP Technology of Texas Instruments. After the PDA nanoparticles were immobilized, the $3 \mathrm{D}$ structure was incubated in PBS for $2 \mathrm{~h}$ at $37^{\circ} \mathrm{C}$, and then irradiated by ultraviolet for $5 \mathrm{~min}$ to allow the polymerization of PDAs.

Efficiency of detoxification. Neutralization of the haemolytic activity of melittin by PDA nanoparticles was assayed by a modified standard haemolytic assay procedure $^{1,2}$. Melittin (final concentration in RBC suspension was $5 \mu \mathrm{g} \mathrm{ml}^{-1}$ ) was pre-incubated with the same volume of PDA nanoparticles for $30 \mathrm{~min}$ at $37^{\circ} \mathrm{C}$ in PBS. The melittin/NP mixture $(100 \mu \mathrm{l})$ was then added to the RBC solution $(2 \% \mathrm{v} / \mathrm{v}, 100 \mu \mathrm{l})$, followed by incubation at $37^{\circ} \mathrm{C}$ for $30 \mathrm{~min}$. Samples were then centrifuged at $800 \mathrm{~g}$ for $5 \mathrm{~min}$. The release of haemoglobin was measured by bicinchoninic acid kit. Controls for 0 and $100 \%$ neutralization of haemolytic activity consisted of RBCs incubated with $5 \mu \mathrm{gml}$ melittin $\left(A_{0 \%}\right)$ and a RBC suspension with normal saline $\left(A_{100 \%}\right)$, respectively. The percentage of neutralization was calculated according to equation (1):

$$
\text { Neutralization }(\%)=100-\left(A_{\text {sample }}-A_{0 \%}\right) \times\left(A_{100 \%}-A_{0 \%}\right)^{-1} \times 100
$$

To study the neutralization efficacy of our 3D PDA nanoparticle-enabled detoxifier, $50 \mu \mathrm{l}$ of melittin $\left(50 \mu \mathrm{g} \mathrm{ml}^{-1}\right)$ was pre-incubated in the device for $60 \mathrm{~min}$ at $37^{\circ} \mathrm{C}$. Then, the melittin solution was carefully collected by directly pipetting the melittin solution and washing the device with $200 \mu \mathrm{l}$ PBS. The resulting $250 \mu \mathrm{l}$ solution was then added to a $250-\mu \mathrm{l}$ RBC solution $(4 \% \mathrm{v} / \mathrm{v})$, followed by incubation at $37^{\circ} \mathrm{C}$ for $30 \mathrm{~min}$. Finally, the percentage of neutralization was calculated. In addition, the neutralization efficiency of the $3 \mathrm{D}$ detoxifier was compared with that of the equivalent amount of PDA nanoparticles or PEGDA hydrogel.

Moreover, the dynamic test was performed on the neutralization efficiency with comparison between the liver-mimetic structure (3D structure) and slab control (that is, with the same total volume). After the incubation of melittin solution with detoxifier for different times, the neutralization efficiency was tested by the haemolytic assay.

\section{References}

1. Hoshino, Y. et al. The rational design of a synthetic polymer nanoparticle that neutralizes a toxic peptide in vivo. Proc. Natl Acad. Sci. USA 109, 33-38 (2012).

2. Hu, C.-M. J., Fang, R. H., Copp, J., Luk, B. T. \& Zhang, L. A biomimetic nanosponge that absorbs pore-forming toxins. Nat. Nanotechnol. 8, 336-340 (2013).

3. Liu, Y. et al. Biomimetic enzyme nanocomplexes and their use as antidotes and preventive measures for alcohol intoxication. Nat. Nanotechnol. 8, 187-192 (2013).

4. Hoshino, Y. et al. Design of synthetic polymer nanoparticles that capture and neutralize a toxic peptide. Small 5, 1562-1568 (2009).

5. Graham, L. M., Nguyen, T. M. \& Lee, S. B. Nanodetoxification: emerging role of nanomaterials in drug intoxication treatment. Nanomedicine 6, 921-928 (2011).

6. Leroux, J.-C. Injectable nanocarriers for biodetoxification. Nat. Nanotechnol. 2, 679-684 (2007).

7. Los, F. C., Randis, T. M., Aroian, R. V. \& Ratner, A. J. Role of pore-forming toxins in bacterial infectious diseases. Microbiol. Mol. Biol. Rev. 77, 173-207 (2013).

8. Gold, B. S., Dart, R. C. \& Barish, R. A. Bites of venomous snakes. New Engl. J. Med. 347, 347-356 (2002).

9. Wardenburg, J. B., Bae, T., Otto, M., DeLeo, F. R. \& Schneewind, O. Poring over pores: $\alpha$-hemolysin and Panton-Valentine leukocidin in Staphylococcus aureus pneumonia. Nat. Med. 13, 1405-1406 (2007).

10. Venkatesan, C. et al. Neutralization of cobra venom by cocktail antiserum against venom proteins of cobra (Naja naja naja). Biologicals 42, 8-21 (2014).

11. Chen, Z. et al. Potent neutralization of anthrax edema toxin by a humanized monoclonal antibody that competes with calmodulin for edema factor binding. Proc. Natl Acad. Sci. USA 106, 13487-13492 (2009).

12. Hung, D. T., Shakhnovich, E. A., Pierson, E. \& Mekalanos, J. J. Small-molecule inhibitor of Vibrio cholerae virulence and intestinal colonization. Science 310, 670-674 (2005).

13. Bayley, H. Membrane-protein structure: Piercing insights. Nature 459, 651-652 (2009).

14. Nel, A., Xia, T., Mädler, L. \& Li, N. Toxic potential of materials at the nanolevel. Science 311, 622-627 (2006).

15. Evenepoel, P. et al. Prometheus versus molecular adsorbents recirculating system: comparison of efficiency in two different liver detoxification devices. Artif. Organs 30, 276-284 (2006).

16. Vignolini, S. et al. A 3D optical metamaterial made by self-assembly. $A d v$. Mater. 24, OP23-OP27 (2012).

17. Tripisciano, C. et al. Activation-dependent adsorption of cytokines and toxins related to liver failure to carbon beads. Biomacromolecules 12, 3733-3740 (2011).

18. Wang, G., Shi, G., Wang, H., Zhang, Q. \& Li, Y. In situ functionalization of stable 3D nest-like networks in confined channels for microfluidic enrichment and detection. Adv. Funct. Mater. 24, 1017-1026 (2014).

19. Zorlutuna, P. et al. Microfabricated biomaterials for engineering $3 \mathrm{D}$ tissues Adv. Mater. 24, 1782-1804 (2012).

20. Zhang, A. P. et al. Rapid fabrication of complex 3D extracellular microenvironments by dynamic optical projection stereolithography. Adv Mater. 24, 4266-4270 (2012).

21. Grogan, S. P. et al. Digital-micromirror-device projection printing system for meniscus tissue engineering. Acta Biomater. 9, 7218-7226 (2013).

22. Qu, X. et al. Relative impact of uniaxial alignment vs form-induced stress on differentiation of human adipose derived stem cells. Biomaterials 34, 9812-9818 (2013).

23. Gou, M. et al. Time-temperature chromatic sensor based on polydiacetylene (PDA) vesicle and amphiphilic copolymer. Sensor. Actuat. B Chem. 150, 406-411 (2010).

24. Lee, S. W. et al. The development of a generic bioanalytical matrix using polydiacetylenes. Adv. Funct. Mater. 17, 2038-2044 (2007).

25. Oya, T. et al. Reversible molecular adsorption based on multiple-point interaction by shrinkable gels. Science 286, 1543-1545 (1999).

26. Li, Y., Yang, H.-H., You, Q.-H., Zhuang, Z.-X. \& Wang, X.-R. Protein recognition via surface molecularly imprinted polymer nanowires. Anal. Chem. 78, 317-320 (2006).

27. Lu, Y. et al. Self-assembly of mesoscopically ordered chromatic polydiacetylene/ silica nanocomposites. Nature 410, 913-917 (2001). 
28. Lee, J. et al. A protective layer approach to solvatochromic sensors. Nat. Commum. 4, 2461 (2013).

29. Wang, X. et al. Colorimetric sensor based on self-assembled polydiacetylene/ graphene-stacked composite film for vapor-phase volatile organic compounds. Adv. Funct. Mater. 23, 6044-6050 (2013).

30. Peng, H. et al. Electrochromatic carbon nanotube/polydiacetylene nanocomposite fibres. Nat. Nanotechnol. 4, 738-741 (2009).

31. Chen, X., Zhou, G., Peng, X. \& Yoon, J. Biosensors and chemosensors based on the optical responses of polydiacetylenes. Chem. Soc. Rev. 41, 4610-4630 (2012).

32. Yarimaga, O., Jaworski, J., Yoon, B. \& Kim, J.-M. Polydiacetylenes: supramolecular smart materials with a structural hierarchy for sensing, imaging and display applications. Chem. Commun. 48, 2469-2485 (2012).

33. Jelinek, R. \& Ritenberg, M. Polydiacetylenes-recent molecular advances and applications. RSC Adv. 3, 21192-21201 (2013).

34. Yoon, B., Lee, S. \& Kim, J.-M. Recent conceptual and technological advances in polydiacetylene-based supramolecular chemosensors. Chem. Soc. Rev. 38, 1958-1968 (2009).

35. Kolusheva, S. et al. Array-based disease diagnostics using lipid/polydiacetylene vesicles encapsulated in a sol-gel matrix. Anal. Chem. 84, 5925-5931 (2012).

36. Dobrovolskaia, M. A. \& McNeil, S. E. Immunological properties of engineered nanomaterials. Nat. Nanotechnol. 2, 469-478 (2007).

37. Gou, M. et al. Curcumin-loaded biodegradable polymeric micelles for colon cancer therapy in vitro and in vivo. Nanoscale 3, 1558-1567 (2011).

38. Miller, J. S. et al. Rapid casting of patterned vascular networks for perfusable engineered three-dimensional tissues. Nat. Mater. 11, 768-774 (2012).

39. Ashley, G. W., Henise, J., Reid, R. \& Santi, D. V. Hydrogel drug delivery system with predictable and tunable drug release and degradation rates. Proc. Natl Acad. Sci. USA 110, 2318-2323 (2013).

40. Qu, X. et al. Regulation of smooth muscle cell phenotype by glycosaminoglycan identity. Acta Biomater. 7, 1031-1039 (2011).

41. Zaman, M. H. The role of engineering approaches in analysing cancer invasion and metastasis. Nat. Rev. Cancer 13, 596-603 (2013).

42. Seliktar, D. Designing cell-compatible hydrogels for biomedical applications. Science 336, 1124-1128 (2012).

43. Kirschner, C. M. \& Anseth, K. S. Hydrogels in healthcare: from static to dynamic material microenvironments. Acta Mater. 61, 931-944 (2013).

44. Heo, Y. J. \& Takeuchi, S. Towards smart tattoos: implantable biosensors for continuous glucose monitoring. Adv. Healthc. Mater. 2, 43-56 (2013).

45. Eichhorn, T. et al. Macroporous composite cryogels with embedded polystyrene divinylbenzene microparticles for the adsorption of toxic metabolites from blood. J. Chem. 2013, 348412 (2013).

46. Xiao, L., Isner, A. B., Hilt, J. Z. \& Bhattacharyya, D. Temperature responsive hydrogel with reactive nanoparticles. J. Appl. Polym. Sci. 128, 1804-1814 (2012).
47. Berezhna, L. G. et al. Structure and biocompatibility of poly (vinyl alcohol)based and agarose-based monolithic composites with embedded divinylbenzene-styrene polymeric particles. Prog. Biomater. 2, 1-8 (2013).

48. Villar, G., Graham, A. D. \& Bayley, H. A tissue-like printed material. Science 340, 48-52 (2013).

49. Mark, A. G., Gibbs, J. G., Lee, T.-C. \& Fischer, P. Hybrid nanocolloids with programmed three-dimensional shape and material composition. Nat. Mater. 12, 802-807 (2013).

50. Taub, R. Liver regeneration: from myth to mechanism. Nat. Rev. Mol. Cell Biol. 5, 836-847 (2004).

\section{Acknowledgements}

The project was supported in part by Grants (EB012597, EB017876 and EY021374) from the National Institute of Biomedical Imaging and Bioengineering and a Grant (CMMI1120795) from the US National Science Foundation to S.C and K.Z.; M.G. and Y.W. appreciated the support from the National Natural Science Foundation (81201785), the National Science and Technology Major Project (2013ZX09301-304-008) and 863 Program (2014AA020509, 2012AA021004). The UCSD Neuroscience Microscopy Shared Facility was supported by Grant P30 (NS047101). We also thank John Warner and Kolin Hribar for their help in preparing the manuscript.

\section{Author contributions}

M.G., X.Q. and S.C. designed the experiment; M.G., X.Q., W.Z. and J.Y. carried out experiments; M.X. performed the computational simulations; and S.C., M.G., Y.W. and K. Z. supervised the project. All co-authors contributed to discussion and analysis of the data.

\section{Additional information}

Supplementary Information accompanies this paper at http://www.nature.com/ naturecommunications.

Competing financial interests: The authors declare no competing financial interests

Reprints and permission information is available online at http://npg.nature.com/ reprintsandpermissions

How to cite this article: Gou, M. et al. Bio-inspired detoxification using 3D-printed hydrogel nanocomposites. Nat. Commun. 5:3774 doi: 10.1038/ncomms4774 (2014).

(c) (1) (2) This work is licensed under a Creative Commons AttributionCY NC SA NonCommercial-ShareAlike 3.0 Unported License. The images or other third party material in this article are included in the article's Creative Commons license, unless indicated otherwise in the credit line; if the material is not included under the Creative Commons license, users will need to obtain permission from the license holder to reproduce the material. To view a copy of this license, visit http:// creativecommons.org/licenses/by-nc-sa/3.0/ 